\title{
Adaptive Photoreceptor with Wide Dynamic Range
}

\author{
Tobi Delbrück \& Carver A. Mead \\ Dept. of Computation and Neural Systems \\ California Institute of Technology \\ Pasadena CA 91125 U.S.A. \\ tdelbruck@caltech.edu
}

\begin{abstract}
We describe a photoreceptor circuit that can be used in massively parallel analog VLSI silicon chips, in conjunction with other local circuits, to perform initial analog visual information processing. The receptor provides a continuoustime output that has low gain for static signals (including circuit mismatches), and high gain for transient signals that are centered around the adaptation point. The response is logarithmic, which makes the response to a fixed image contrast invariant to absolute light intensity. The 5-transistor receptor can be fabricated in an area of about $70 \mu \mathrm{m}$ by $70 \mu \mathrm{m}$ in a $2-\mu \mathrm{m}$ single-poly CMOS technology. It has a dynamic range of 1-2 decades at a single adaptation level, and a total dynamic range of more than 6 decades. Several technical improvements in the circuit yield an additional 1-2 decades dynamic range over previous designs without sacrificing signal quality. The lower limit of the dynamic range, defined arbitrarily as the illuminance at which the bandwidth of the receptor is $60 \mathrm{~Hz}$, is at approximately 1 lux, which is the border between rod and cone vision and also the limit of current consumer video cameras. The receptor uses an adaptive element that is resistant to excess minority carrier diffusion. The continuous and logarithmic transduction process makes the bandwidth scale with intensity. As a result, the total A.C. RMS receptor noise is constant, independent of intensity. The spectral density of the noise is within a factor of two of pure photon shot noise and varies inversely with intensity. The connection between shot and thermal noise in a system governed by Boltzman statistics is beautifully illustrated.
\end{abstract}

\section{THE CIRCUIT}

A simple nonadaptive source-follower logarithmic receptor is shown on the left of Figure 1. The gain is $\mathrm{V}_{\mathrm{T}}=\mathrm{kT} / \mathrm{q}=25.4 \mathrm{mV}$ per e-fold intensity change. The performance of the source-follower receptor is difficient in two respects: The offsets are as large as signals produced by typical scenes, and the response is too slow.

The adaptive receptor shown on the right of Figure 1 remedies both problems. It consists of a source-follower receptor input stage combined with amplification and lowpass-filtered feedback. A conceptual way of thinking about the operation is indicated by the shaded areas. The circuit uses an internal model to make a prediction about the input signal. The output comes from a comparison of the input and the prediction. The loop is completed by using learning to refine the model

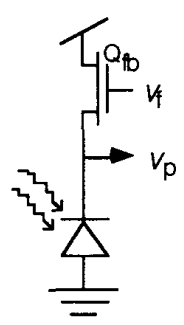

Source-Follower Receptor

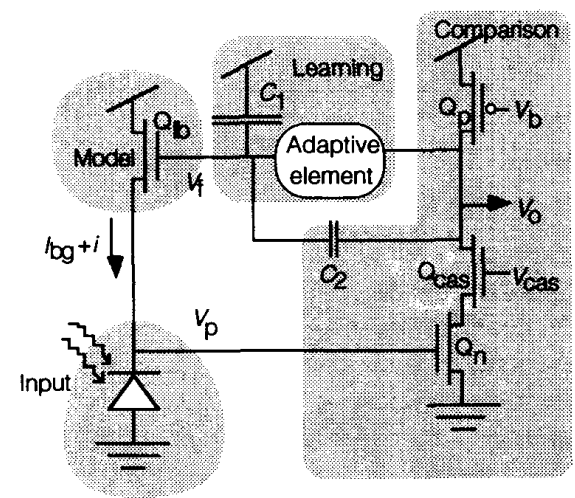

Adaptive Receptor
FIGURE 1 Receptor circuits.

so that predictions become more accurate. The level adaptation in the adaptive receptor is perhaps the simplest type of learning.

The output of the source-follower receptor is amplified by the inverting amplifier consisting of $Q_{n}, Q_{c a s}$ and $Q_{p}$. The voltage gain $-A_{\mathrm{amp}}$ is typically several hundred. Bias voltage $V_{\mathrm{b}}$ determines the cutoff frequency for the receptor, by setting the bias current in the inverting amplifier. $Q_{c a s}$ is a cascode that nullifies the Miller capacitance from the gate to the drain of $Q_{n}$ and also doubles the gain of the amplifier.

The feedback loop is completed when the output $V_{\mathrm{o}}$ is fed back to $V_{\mathrm{f}}$ through the resistor-like adaptive element and the capacitive divider formed from $C_{1}$ and $C_{2}$.

\section{Receptor Gain}

The high gain in the feedback loop effectively clamps $V_{\mathrm{p}}$. When the input current changes an e-fold, $V_{\mathrm{f}}$ must change by $\mathrm{V}_{\mathrm{T}} / \mathrm{K}$ to hold $V_{\mathrm{p}}$ clamped, where $\kappa \approx 0.8$ is the back-gate coefficient. Hence, the small-signal steady-state gain is given by

$$
\frac{v_{\mathrm{o}} / V_{\mathrm{T}}}{i / I_{\mathrm{bg}}}=\frac{1}{\mathrm{~K}} \quad \text { (steady-state closed-loop gain) }
$$

where $i$ is a small signal variation in the steady-state background intensity $I_{\mathrm{bg}}$, and $v_{\mathrm{o}}$ is a small-signal change in the 
FIGURE 2 Responses of the source-follower and adaptive receptor over 7 decades of background. (The source-follower response is shown inverted.) Stimulus is a square-wave variation in the intensity, centered around a mean value. The numbers by each section are the log intensity of the mean value; $0 \mathrm{log}$ is $2.9 \mathrm{~W} / \mathrm{m}^{2}$, about the level of direct office fluorescent light. The source-follower receptor completely smooths out the square wave input at the lowest intensities, while the adaptive receptor still responds. The $1.8 \mathrm{~Hz}$ square wave stimulus is from a red LED $(635 \mathrm{~nm})$. The irradiance varies by a factor of about 2 at each background level. At the lowest intensity, the bandwidth of the adaptive receptor is about $5 \mathrm{~Hz}$, and about 700 photons are collected during each integration time.

ouptut. For transient signals, where the output must go through the capacitive divider, the gain is

$$
A_{\mathrm{cl}} \equiv \frac{\nu_{\mathrm{o}} / V_{\mathrm{T}}}{i / I_{\mathrm{bg}}}=\frac{1}{\mathrm{\kappa}} \frac{C_{1}+C_{2}}{C_{2}} \quad \text { (transient closed-loop gain) }
$$

We shall call the gain $A_{\mathrm{cl}}$ for transient signals the closed loop gain.

The clamping of the input nodes speeds up the receptor response. The larger the gain $A_{\mathrm{amp}}$ of the feedback amplifier, the less the input node needs to move, and the more speedup we obtain. On the other hand, for a given feedback amplifier, the more closed-loop gain $A_{\mathrm{cl}}$ we design in the receptor (by adjusting the capacitive divider ratio), the more the input node must move, and the slower the response. We shall take as a baseline the speed of the source-follower receptor. The time constant of this receptor is

$$
\tau_{\text {in }}=\frac{C}{g}=\frac{C V_{\mathrm{T}}}{I_{\mathrm{bg}}} \quad \text { (baseline speed) }
$$

The speedup obtained by using the active feedback to clamp the input node is given by

$$
A_{\text {loop }}=\frac{A_{\text {amp }}}{A_{\mathrm{cl}}} \quad \text { (speedup) }
$$

The speedup is equal to the total loop gain, obtained by multiplying the gain all the way around the loop. A typical measured speedup, using the active feedback and the cascode, is 1-2 decades.

The gain-bandwidth product of the adaptive receptor is theoretically larger by a factor of $A_{\text {amp }}$ than that of the sourcefollower receptor. $Q_{\text {cas }}$ nullifies the gate-drain Miller capacitance of $\mathrm{Q}_{\mathrm{n}}$. Another Miller capacitance at the source of $\mathrm{Q}_{\mathrm{fb}}$, although reduced by the capacitive divider, ultimately limits the possible speedup afforded by using higher $A_{\text {amp }}$, particularly with small $A_{\mathrm{cl}}$. In practice, the ratio of the gain-bandwidth products between the source-follower receptor and the adaptive receptor is several hundred.

\section{Response Characteristics}

Figure 2 illustrates the receptor's adaptive behavior and the invariance of the response to absolute intensity. The traces compare the response of the nonadaptive source-follower receptor with the response of the adaptive receptor. The incident signal is a small intensity variation sitting on a steady

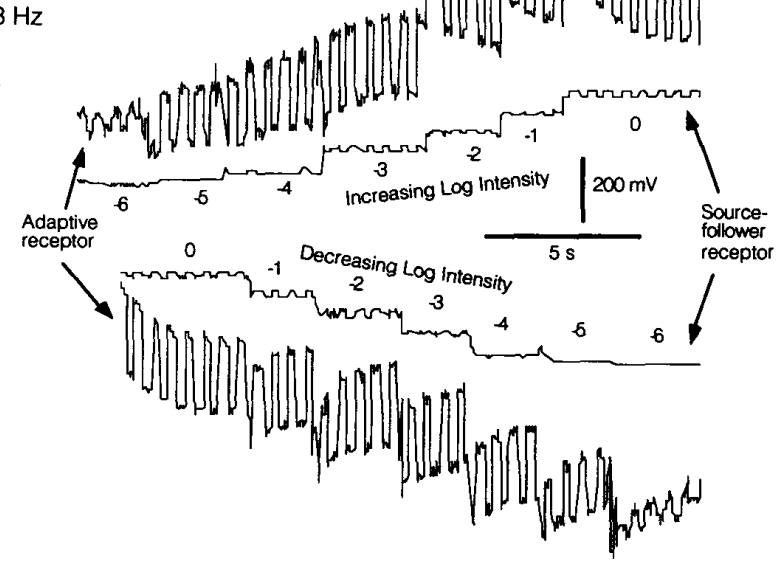

background. The small variations represent the type of signal arising from objects in a real scene, while the steady background represents the ambient lighting level. The contrast of the signal is a fixed percentage, as would be produced by reflective objects. We varied the overall intensity level by interposing neutral density filters with various attenuation factors between the light source and the receptor to simulate changes in the ambient lighting, as would be caused by passing from shadow into sunlight or vice versa.

The amplitude of the response to the small contrast variation is almost invariant to the absolute intensity, owing to the logarithmic response property. The adaptation makes the receptor have high gain for rapidly varying intensities and low gain for slowly varying intensities. A careful examination shows that the receptor adapts very rapidly in response to the decade changes in intensity. This rapid adaptation is due to the use of an adaptive element with an expansive nonlinearity.

\section{ADAPTIVE ELEMENT}

Adaptation occurs when charge is transferred through the adaptive element onto or off the storage capacitor. The adaptive-element is a resistor-like device that has a monotonic I$\mathrm{V}$ relationship. (True ohmic resistors available in a gardenvariety CMOS fabrication process are much too small in value for adaptation on the time scale of seconds.) The adaptive element acts like a pair of diodes, in parallel, with opposite polarity. The current increases exponentially with voltage for either sign of voltage, and there is an extremely high-resistance region around the origin, as shown in Figure 4.

The I-V relationship of the expansive element means that the effective resistance of the element is huge for small signals and small for large signals. Hence, the adaptation is 

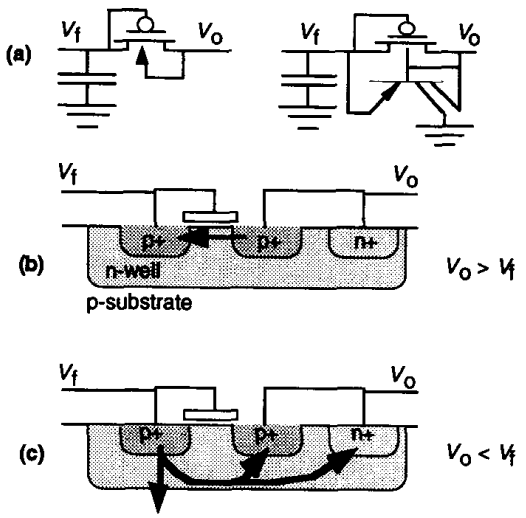

slow for small signals and fast for large signals. This behavior is useful, because it means that the receptor can adapt quickly to a large change in conditions-say, moving from shadow into sunlight-while maintaining high sensitivity to small and slowly varying signals.

For voltage polarity $V_{\mathrm{o}}>V_{\mathrm{f}}$ across the element, the MOS transistor is turned on and the bipolar transistor is turned off. The driven side $\left(V_{0}\right)$ in the MOS case acts as the source of the transistor, but because the back gate (the well) is driven at the same time, the current e-folds every $V_{\mathrm{T}} / \kappa$.

For opposite polarity, the MOS transistor is turned off and the bipolar is turned on. The driven side forward-biases the $\mathrm{p}+/ \mathrm{n}$ emitter-base junction. The bipolar transistor has two collectors, the driven side and the substrate. The current efolds every $V_{\mathrm{T}}$ volts.

Detailed measurements indicate that the conductance of the element at zero differential voltage is approximately equal to $(0.1-1 \mathrm{fA}) / \mathrm{V}_{\mathrm{T}}$. Photocurrent in the emitter-base junction by scattered light increases the conductance proportionally to the intensity for irradiances greater than approximately $100 \mathrm{~mW} / \mathrm{m}^{2}$. A key feature of this adaptive element is that the currents in the capacitor node $\left(V_{\mathrm{f}}\right)$ are unaffected by light-generated minority carriers, unlike many other device structures.

\section{Second-order temporal effects}

A second-order time-domain analysis reveals that in order for the receptor to be a first-order system (i.e. produce a nonringing response to a step input), the output-node time constant of the feedback amplifier must be faster than the inputnode time constant by a factor of $A_{\text {loop }}$. However, since the maximum $Q$ of the circuit only scales as $\sqrt{A_{\text {loop }}}$, a large bias current may not be essential. We usually tolerate some resonance in return for filtering out flicker from artificial lighting and reducing noise at high intensities.
FIGURE 4 Measured current-voltage relationship for the adaptive element. The bipolar mode conduction efolds every $28 \mathrm{mV}$, compared with $48 \mathrm{mV}$ for the MOS mode.

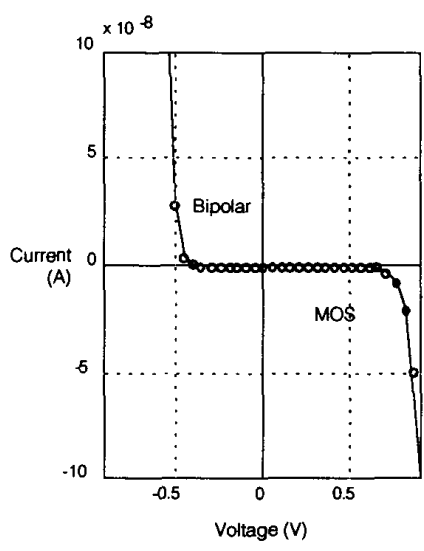

\section{Dynamic Range}

The usable bandwidth of the receptor is proportional to the intensity. The receptor has a bandwidth of at least $60 \mathrm{~Hz}$ (like human photopic vision) down to an irradiance of about $1 \mathrm{~mW} / \mathrm{m} 2$. This irradiance corresponds to an illuminance of about 1 lux for $555 \mathrm{~nm}$ (yellow) photons.

\section{NOISE}

An interesting and important feature of logarithmic receptors is that the bandwidth scales with intensity. The shot noise spectral power density scales inversely with intensity, so the total RMS receptor noise is a constant that depends only on the effective receptor input capacitance. The equipartition principle from statistical mechanics tells us that the total charge fluctuation on the input node is given by $\Delta Q^{2}=k T C_{\text {eff }}$, where $C_{\text {eff }}$ is the effective input capacitance including the effect of total loop gain. The charge fluctuations result in voltage fluctuations that are interpreted as current fluctuations. The total receptor noise in dimensionless input-referred units is given by

$$
\frac{\Delta i^{2}}{I_{b g}^{2}}=\frac{q}{C_{\text {eff }} V_{\mathrm{T}}} \quad \text { (total noise) }
$$

For a typical input capacitance of $100 \mathrm{fF}$ and a total loop gain of 30 , the total noise is $0.2 \%$. The spectral density of the noise within the passband is given by a simple computation involving the first-order low-pass filter at the input:

$$
S=\frac{4 q}{I_{\mathrm{bg}}} \quad \text { (noise spectral density) }
$$

$S$ is the shot noise power measured in the input-referred units per unit frequency $(\mathrm{Hz})$. It is nearly the time per unit charge, which makes sense. At an irradiance of $1 \mathrm{~mW} / \mathrm{m}^{2}$ (moonlight), with a $20 \mu \mathrm{m}$ by $20 \mu \mathrm{m}$ diode, $S=3 \mu \mathrm{s}$, meaning that if the receptor had a bandwidth of $\mathrm{MHz}$, the total noise 

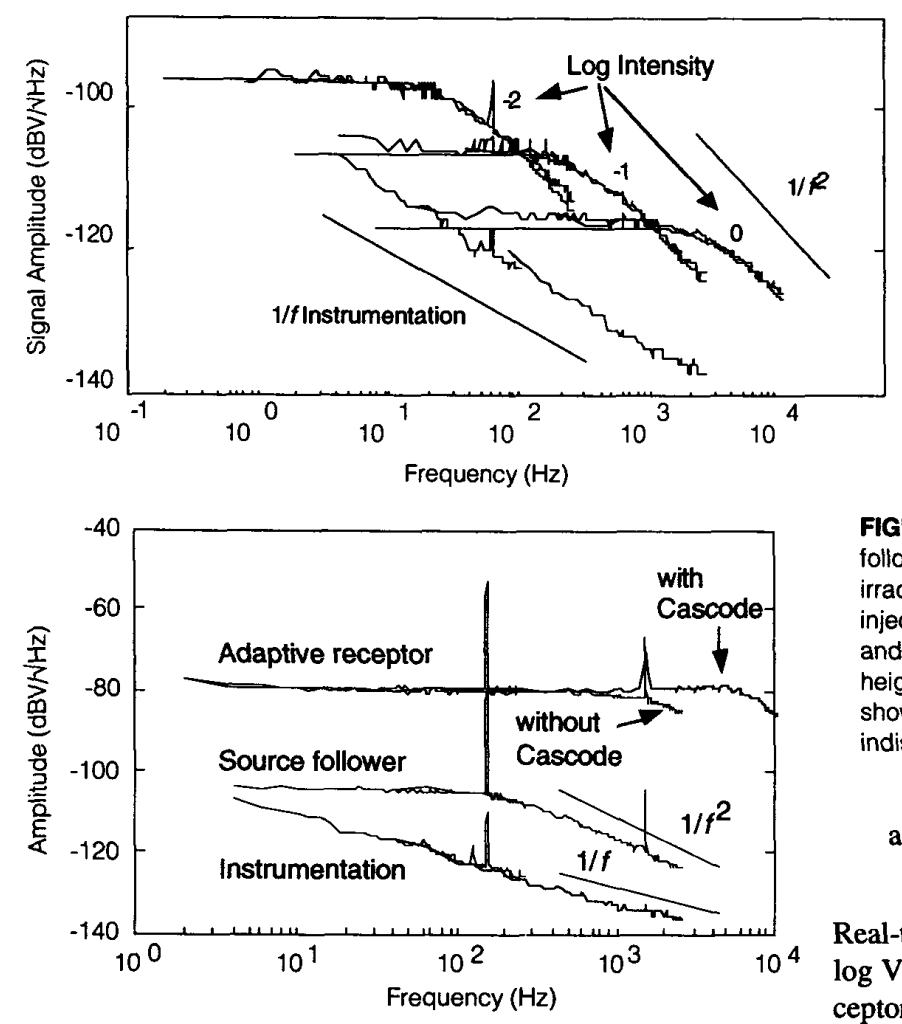

be unity. Since the bandwidth is not $\mathrm{MHz}$, the total noise is scaled down proportionately.

Figure 5 shows how the bandwidth trades off against the noise spectral density. The smooth curves are the theoretical fits. The receptor noise is very close to pure photon shot noise, since the quantum efficiency of the junction is at least 0.5 in the visible. Figure 6 shows the noise behavior of the adaptive receptor with and without the cascode, relative to the source-follower receptor. The SNR of the adaptive receptor is within a factor of two of the SNR of the source-follower receptor.

\section{TECHNICAL INNOVATIONS}

There are three technical improvements in the work here compared with previously published photoreceptor designs:

1. Using a photodiode, instead of the phototransistor used in previous designs, increases the dynamic range by at least 1 decade, without degrading signal quality.

2. Using a single-transistor cascode in the feedback amplifier, yields another 0.5 decade dynamic range.

3. Using the new adaptive element removes a large steadystate offset voltage and increases the adaptation time constant and the symmetry of the receptor response to light- and dark-going transients. Previous designs used elements that were very susceptible to the effects of par-
FIGURE 5 Noise spectra for the sourcefollower receptor. The curves show the intrinsic noise spectra from the receptor at a given level of steady background intensity. The number by each curve is the log background irradiance; 0 log irradiance is $1.7 \mathrm{~W} / \mathrm{m}^{2}$. The smooth curve is the theoretical fit. Definition of $\mathrm{dBV}$ units is the signal power, in $\mathrm{dB}$, relative to a $1 \mathrm{~V}$ signal.

FIGURE 6 Comparison of noise spectra for simple source follower receptor and the adaptive receptor. The DC irradiance for this measurement is $0.2 \mathrm{~W} / \mathrm{m}^{2}$, and the injected signal is a combination of a $150 \mathrm{~Hz}$ sinusoidal signal and a $1.5 \mathrm{kHz}$ signal each with contrast of about $1 \%$. The height of the signal spike relative to the surrounding noise shows that the SNR for the receptors are nearly indistinguishable.

asitic minority carriers.

\section{DISCUSSION}

Real-time spatiotemporal computations fit naturally to ana$\log$ VLSI. We have shown how to build a practical photoreceptor circuit that is suitable as a front-end transducer for analog VLSI vision systems. The receptor is usable under photopic vision conditions. The continuous transduction process leads to intensity-invariant total noise that is within a factor of two of photon counting noise. Photoreceptors of this type will undoubtedly be used in commercial vision systems, where designers will not be able to resist the capability to link low-power, continuous-time computation together with transduction.

\section{Acknowledgments}

We acknowledge support from the ONR and MOSIS. Rahul Sarpeshkar made a crucial observation about the relationship between shot and thermal noise. Shih-Chii Liu, David Standley, and Dick Lyon provided helpful comments about this work.

\section{Further Information}

A complete list of references, along with a detailed description of the circuit behavior and other topics in analog VLSI phototransduction, is available via anonymous $\mathrm{ftp}$ to hobiecat.pcmp.caltech.edu, in the directory $\sim f t p / p u b / a n a p r o s e / t o b i$. (Send mail to tdelbruck@caltech.edu for help.) 